\title{
Adoption of the Co-Occurrence Matrix and Artificial Neural Networks in Fingerprint Recognition
}

\section{Maysoon Khidr Al-Nuaimi}

\section{College of Computer Science and Mathematics}

University of Mosul, Mosul, Iraq

Received on: 26/09/2012

Accepted on: 30/01/2013

\section{ABSTRACT}

This paper presents a new method for fingerprint recognition depending on various sizes of fingerprint images. The proposed algorithm applied on more than 30 fingerprint samples, the results was good.

The proposed algorithm begins with apply enhancement operations on the fingerprint image to eliminate unwanted noise around the fingerprint by using median filter. Then apply thinning operation on the enhanced image and compute co-occurrence matrices for produced image. Next, the properties of the co-occurrence matrices used as inputs of the neural network for recognition process. To speed the recognition process back propagation network used. The ratio of recognition about $100 \%$.

Keywords: Artificial Neural Networks, Fingerprint, Recognition.

$$
\begin{aligned}
& \text { اعتماد مصفوفة التواجد والثبكات العصبية الاصطناعية في تمييز بصمة الأصبع } \\
& \text { ميسون خضر النعيمي } \\
& \text { كلية علوم الحاسوب والرياضيات } \\
& \text { جامعة الموصل } \\
& \text { تاريخ قبول البحث: 2013/01/30 } \\
& \text { تاريخ استلام البحث: 2012/09/26 } \\
& \text { الملخص } \\
& \text { البصمة وتم تطبيقها على أكثر من } 30 \text { عينة لصور بصمات الأصابع وقد تم التوصل إلى نتائج جيدة. }
\end{aligned}
$$

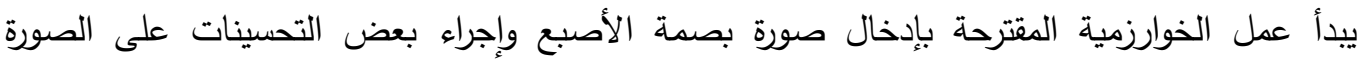

$$
\begin{aligned}
& \text { اللتخلص من الضوضاء غير المرغوب فيها والتي توجد على حدود البصمة باعتماد الفلتر المتوسط ( median } \\
& \text { (filter وقراءتها بوصفها مصفوفة ثنائية رقمية ومن ثم تتحيف الصورة وإيجاد مصفوفة التواجد للصورة المحسنة } \\
& \text { والتي أجريت عليها عملية التحيف وإدخال الصفات التابعة لهذه المصفوفة على الثبكة العصبية لغرض التمييز . } \\
& \text { تم توظيف شبكة الانتشار العكسي (Back propagation) من اجل تسريع عملية التمييز وقد كانت نسبة التمييز } \\
& \% 100 \\
& \text { الكلمات المفتاحية: مصفوفة التواجد، الثبكات العصبية الاصطناعية، التمييز، بصمة الأصبع. }
\end{aligned}
$$

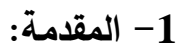


إن تمييز الأنماط هو أحد فروع علم معالجة الصور إذ يهدف إلى إيجاد أو تطوير تقنيات للتعرف على إلى

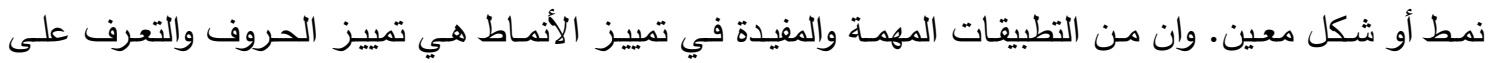
الأشكال والأشخاص وكذلك يستخدم في المجالات الطبية. إن تحليل نسيج الصورة واستخلاص أهم الخواص للصورة من الخطوات الرئيسة المتبعة لغرض تمييز الأنماط وعلى الرغم من وجود العديد من الطرائق المستخدمة لتحليل نسيج الصورة مثل استخدام الشبكات العصبية

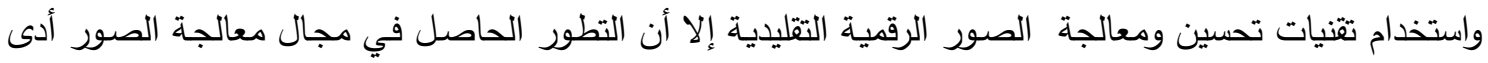

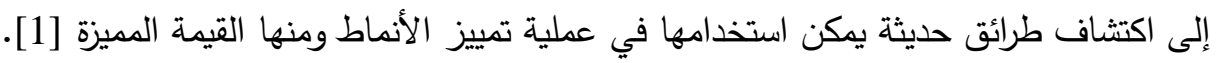

2- تمييز بصمات الأصابع

عرف ألإنسان بصمات ألأصابع والكف وباطن القدم منذ عصر ما قبل التاريخ، وقد أكد الباحثون والعلماء ذلك من خلال ألآثار التي عثروا عليها في مختلف أرجاء العالم وذكر بشكل أو بآخر من خلال الكتب السماوية وندان المقدسة إذ يحكى أن بطرس الرسول قد وقّح كتابه ببصمة إصبع [2][3]، وتعد بصمات الأصـابع والكف وباطن

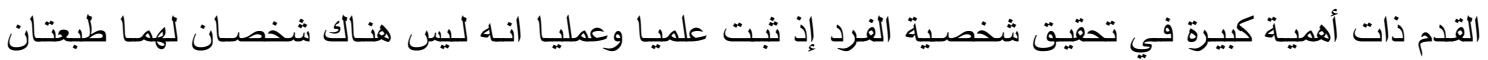
متطابقتان لإصبعين في الخطوط والمميزات حتى لو كان الثخصـان توأمين، ولم يحدث إلى حد ألان أن هناك

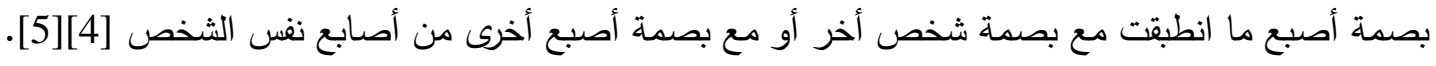
3- 3 شكل بصمة الإصبع العام

يلاحظ الثكل العام لبصمة الإصبع إن سطحه مكسو بخطوط دقيقة متوازيـة ترتقع عن سطح جلد البشرة

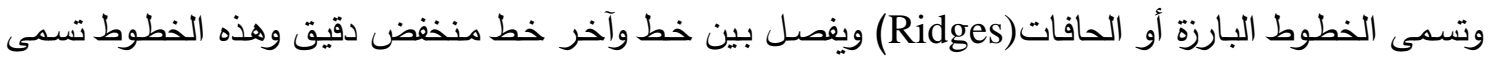

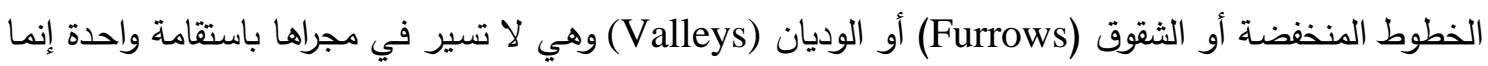
تكوّن أشكالا متتوعة عديدة غريبة في ترتيبها فمنها تكون على شكل دوائر متعددة حول نقطة الوسط بينما غيرها تكون على هيئة خطوط منحدرة إلى اليمين أو اليسار ، وهكذا تتكون منها خطوط مقوسة تبدأ من جهة وتنتهي من

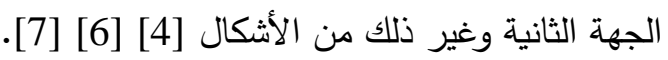
أن كل شخص يمتلك خصائص فسلجية وسلوكية معينة خاصة به، وتعد بصمة الأصبع واحدة من أكثر

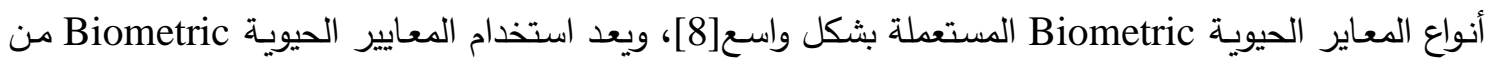
احد أهم المقاييس المستخدمة في الكثف عن هوية الأشخاص. وقد استخدم البابليون الأوائل بصمة اليد في الطين لإثبات الملكية (بوصفها توقيعا) [9]. والثكل (1) يوضح الثكل العام لبصمة الأصبع. 


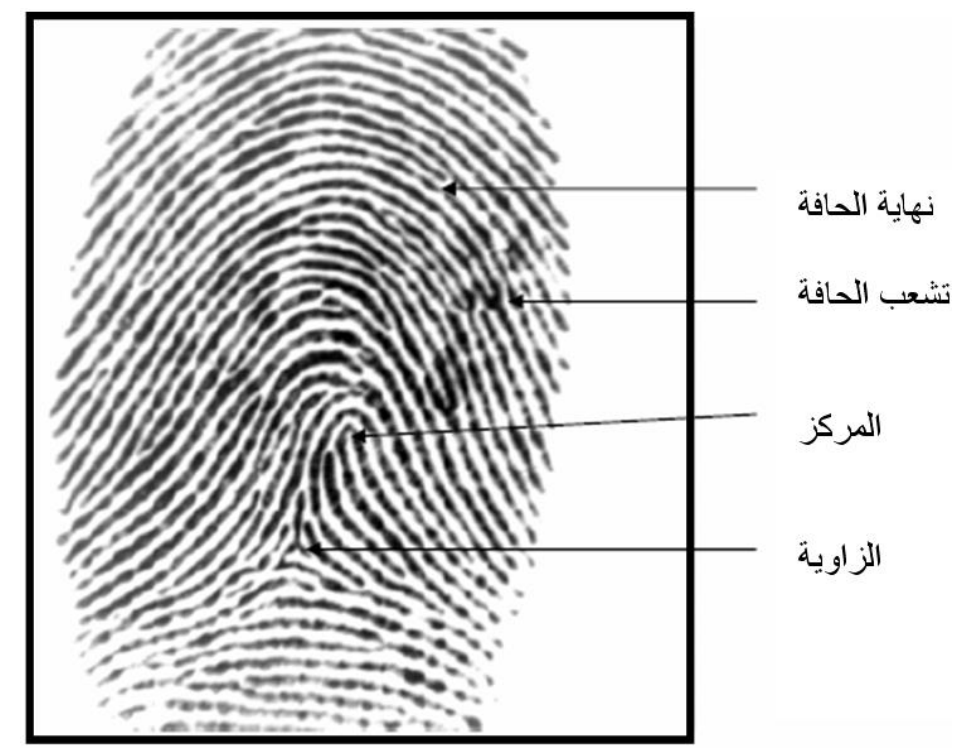

$$
\text { الثكل (1). الثكل العام لبصمة الإصبع }
$$

ولغرض استخدام أجزاء من الخواص الجزئية معايير حيوية لابد من توفر الثروط الأربعة الآتية:

أ- الثمولية Universality

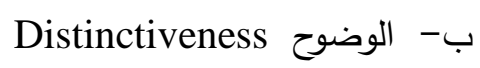
ت- ت الدوام الوضوح

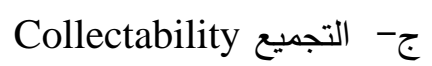
وهناك أنواع للبصمات تكون على نوعين:

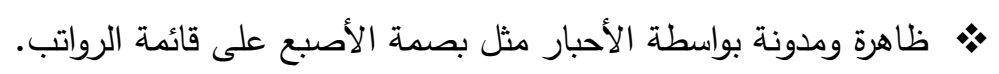

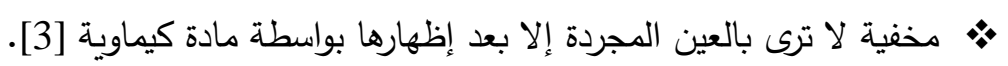

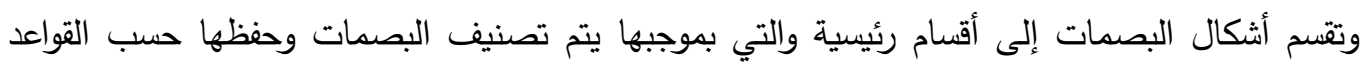
الفنية التي وضعها (A. R. Henry) وتعد هذه الطريقة عالمية وتستعمل في أكثر دول العالم، وبموجب هذه ونئ الطريقة تقسم بصمة الإصبع إلى الإثكال والثروع الآتية [3] [4: الطعدة:

$$
\begin{aligned}
& \text { • Arches المقوسات } \\
& \text { • • • • • } \\
& \text { • • • • المستديرات ل }
\end{aligned}
$$

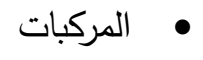

$$
\begin{aligned}
& \text { • المتوعات المركات }
\end{aligned}
$$

411][10] Co-occurrence Matrices مصفوفات حدوث المشاركة

إن هذه المصفوفات اقترحها العالم haralick في عام 1973، وهي مصفوفة ثنائية الأبعاد (معتمدة على المستويات الرمادية)، تستخدم بثكل أساسي في تحليل النسجة بسبب قدرتها العالية على تحديد الترابط المكاني لقيم المستويات الرمادية في الصورة، إذ تعمل مصفوفة حدوث المشاركة (P) بوصفها جامعاً تراكميا (accumulator)

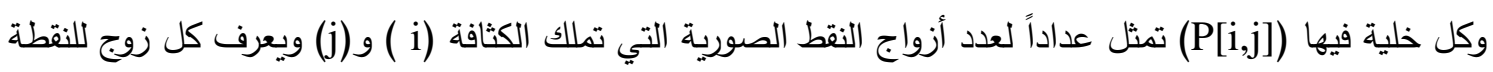


الصورية بالبعد والاتجاه الذي يمكن أن يمثل بمتجه الإزاحة (dx,dy)(d)، حيث أن (dx) يمثل إزاحة النقطة

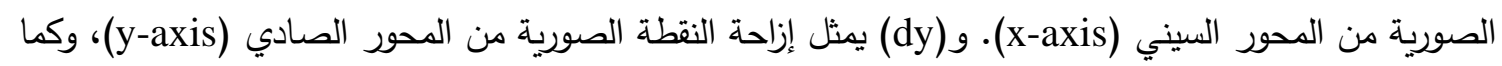

موضح في الثكل (2).

\begin{tabular}{|c|c|c|c|c|c|c|c|c|c|c|c|c|c|c|}
\hline & & & & & & & & 2 & 3 & 4 & 5 & 6 & 7 & \\
\hline 1 & 1) & 5 & 6 & 8 & GLCM & 1 & 1 & 2 & 0 & 0 & 1 & 0 & 0 & 0 \\
\hline 2 & 3 & 5 & 7 & 1 & & 2 & 0 & $r_{0}$ & 1 & 0 & 1 & 0 & 0 & 0 \\
\hline 4 & 5 & 71 & 1 & 2 & & 3 & 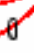 & 0 & 0 & 0 & 1 & 0 & 0 & 0 \\
\hline 8 & 54 & & 2 & 5 & & 4 & 0 & 0 & 0 & 0 & 1 & 0 & 0 & 0 \\
\hline & & & & & & 5 & 1 & 0 & 0 & 0 & 0 & 1 & 2 & 0 \\
\hline & & & & & & 6 & 0 & 0 & 0 & 0 & 0 & 0 & 0 & 1 \\
\hline & & & & & & 7 & 2 & 0 & 0 & 0 & 0 & 0 & 0 & 0 \\
\hline & & & & & & 8 & 0 & 0 & 0 & 0 & 1 & 0 & 0 & 0 \\
\hline
\end{tabular}

الثكل(2). مثال توضيحي لمصفوفة حدوث المشاركة

ومن اجل تحديد الاعتماد المكاني لقيم المستوى المكاني تم حساب عدد من خواص النسجة وأهمها ما

يأتي:-

1- الطاقة Energy تحدد بعدد أزواج النقطات الصورية المكررة ويتوقع بان تكون الطاقة مرتفعة إذا كان هناك $\mathrm{m} \mathrm{n}$

عدد كبير من الأزواج للنقاط الصورية (pixels pair) ، وتحسب كما في المعادلة الآتية:-

Energy $=\Sigma \Sigma p^{2}[i, i]$

$$
\text { حيث: }
$$

P Pصفوفة حدوث المشاركة.

n\&m

2- التباين contrast يتوقع بان يكون التباين في الصورة منخفضا إذا كانت المستويات الرمادية لكل زوج من

النقاط الصورية متساوية فهي مقياس التباين المحلي للصورة، والمعادلة الآتية تبين كيفية حساب التباين:-

Contrast $=(\mathrm{i}-\mathrm{j})^{2} \mathrm{p}[\mathrm{I}, \mathrm{j}]$

3- التجانس homogeneity يتوقع بان يكون التجانس في الصورة مرتفعا إذا كانت المستويات الرمادية لكل

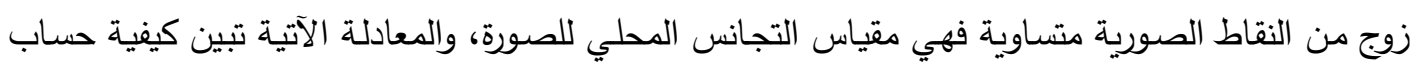

Homogeneity $=\stackrel{\mathrm{m}}{\Sigma} \sum^{\mathrm{n}} \frac{p[i, j]}{1-|i-j|}$ التجانس:-

4- الارتباط correlation يمثل قيمة ارتباط ما بين نقطتين صوريتين في كل زوج، وتكون قيمته اكبر كلما

كانت المستويات الرمادية في زوج النقط الصورية متقاربة، ويحسب معامل الارتباط بالمعادلة الآتية:-

Correlation $=\sum \sum \frac{i-\mu(j-\mu) p[i, j]}{\delta}$ 
5- الثبكات العصبية الاصطناعية وتمييز الأنماط [12]

طورت الثبكات العصبية الاصطناعية ANN لتحقيق أداء يشبه أداء الإنسان في حل بعض المشاكل

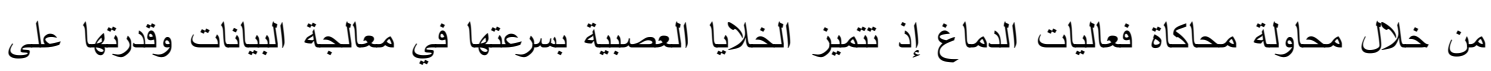
التعلم والتعامل مع أنماط بيانات خاطئة مما جعلها مناسبة لكثير من المجالات التطبيقية مثل تتفيذ بعض المضئل المسائل

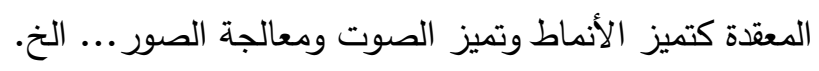

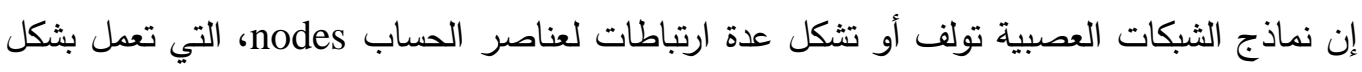

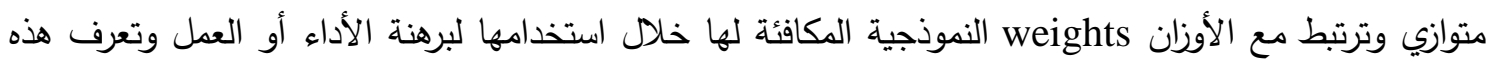

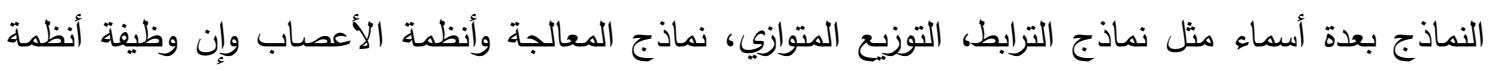
الأعصاب الاصطناعية هي التقيم المتوازي لحسابات الثبكة ومعظم الخصائص الأساسية هي لمعمارية الثبكة

العصبية.

بعض الثبكات تجهز إجابات فورية وبعضها تحتاج إلى وقت للإجابة حسب ما تمتلك من صفات وسلوك ويشار

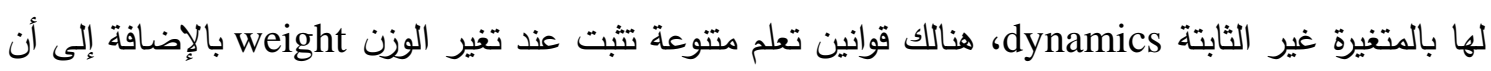

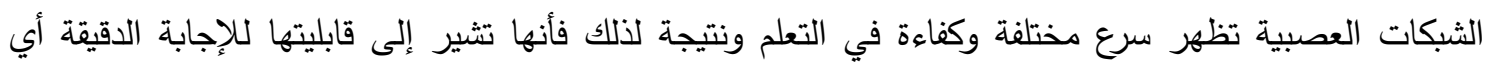
تعرف ماذا سيكون الإخراج لهذا الإدخال وتكون نماذج الشبكات العصبية التي تكثف ونف عدة منافسات متزامنة تستخدم شبكة التوازي الكبيرة التي تتألف من عناصر حسابات nodes مرتبطة بواسطة خط توصيل مع أوزان مختلفة وهناك أنواع متعددة من الشبكات العصبية لكن كلها تحتوي ثلاث أشياء:1- الخلية مode.

2- وحدة المعالجة processing unit وصفتها أنها يوجد ارتباطات فيما بينها (أي ترتبط خلية مـع أخرى عن طريق الأوزان (weights).

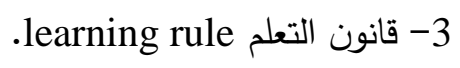

هذه الثلاث هيئات تشكل مع بعضها نموذج الشبكة العصبية. [13]

6 -6ور تدريب الثبكات العصبية الاصطناعية Artificial Neural Networks Training

هو طور في الثبكات العصبية عندما تتتج بيانات جديدة في الثبكة نتيجة تغير الوزن weight للشبكة وان الشبكة تدرب على التطبيق المعطى أي على مجموعة الإدخالات لإنتاج الإخراج المطلوب والتدريب ينجز

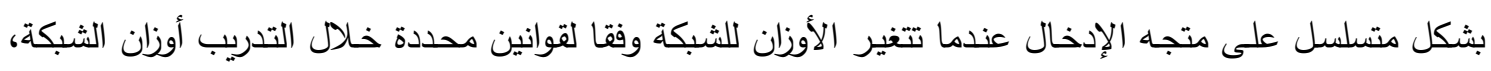

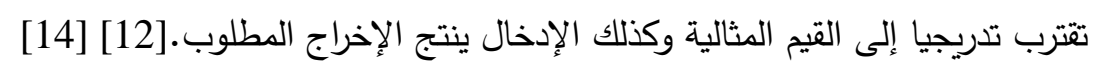
7- الثبكة العصبية ذات الانتشار العكسي BPNN:

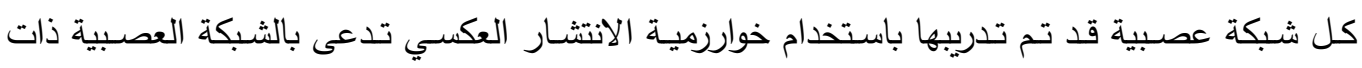

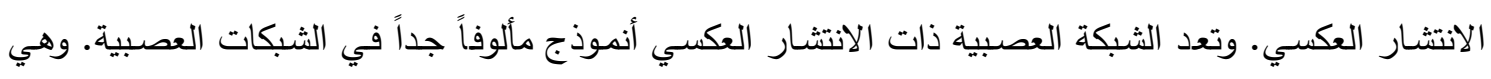

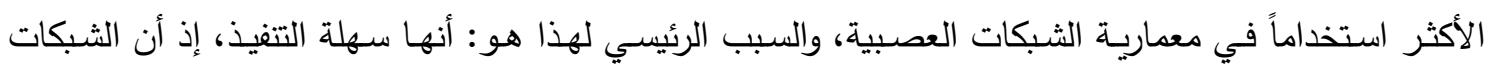

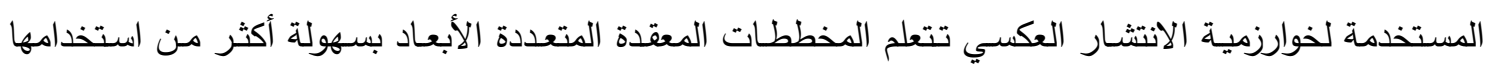

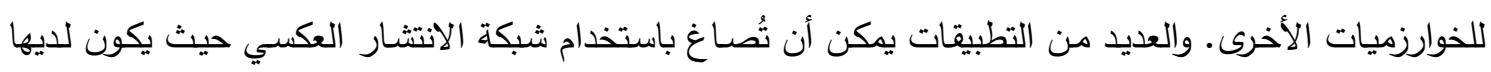


نموذج لأغلب الشبكات العصبية المتعددة الطبقات. ومن ثم يمكننا القول إن أكثر طريقة تدريب شائعة الاستخدام

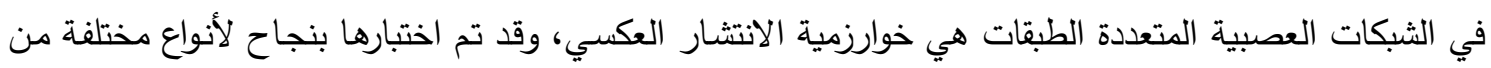

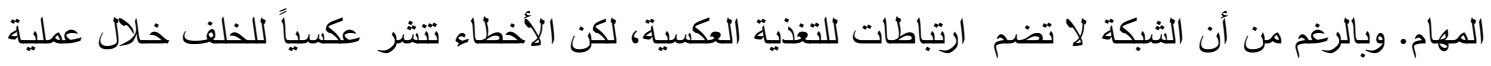
التدريب. والأخطاء في طبقة الإخراج تحدد قياسات الأخطاء في الطبقة المخفية، والتي تستخدم كانحياز لتعديل أوزان الربط بين طبقة الإدخال والطبقة المخفية. كل معالجة تكرار تضم تعديل مجموعتين من الأوزان على الأقل

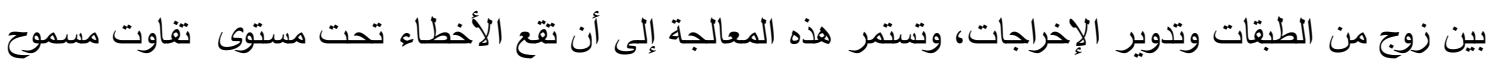

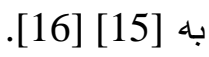

\section{8- معمارية الثبكات العصبية ذات الانتشار العكسي BPNN:}

تعد الثبكة العصبية ذات الانتشار العكسي نظام شبكة بتغذية أمامية وبصورة تدرجية، وتتألف من طبقتين

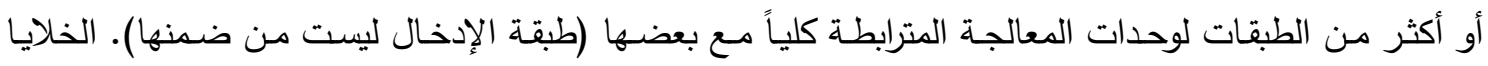

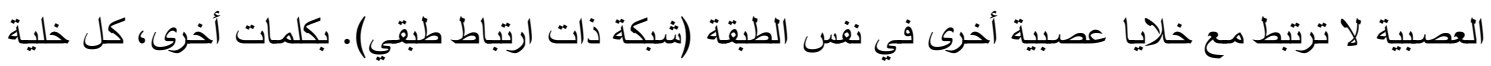

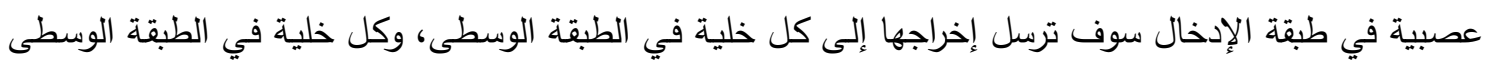
سوف ترسل إخراجها إلى كل خلية في طبقة الإخراج [16] [17] [18]. عدد الخلايا العصبية في الطبقة المخفية

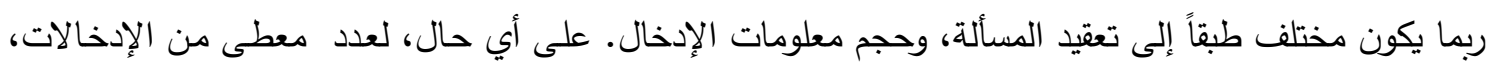

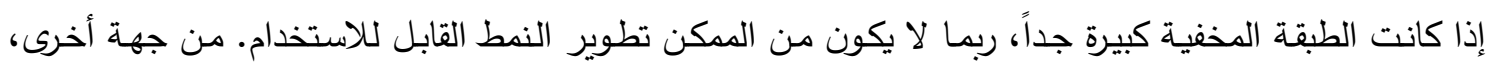

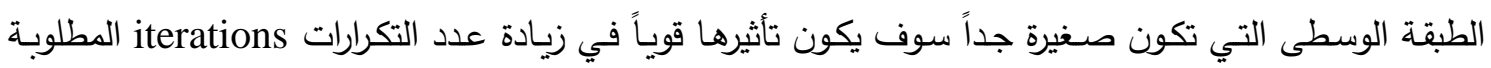
لتدريب الثبكة [17] والثكل (3) يوضح الثكل العام للشبكة العصبية المتعددة الطبقات.

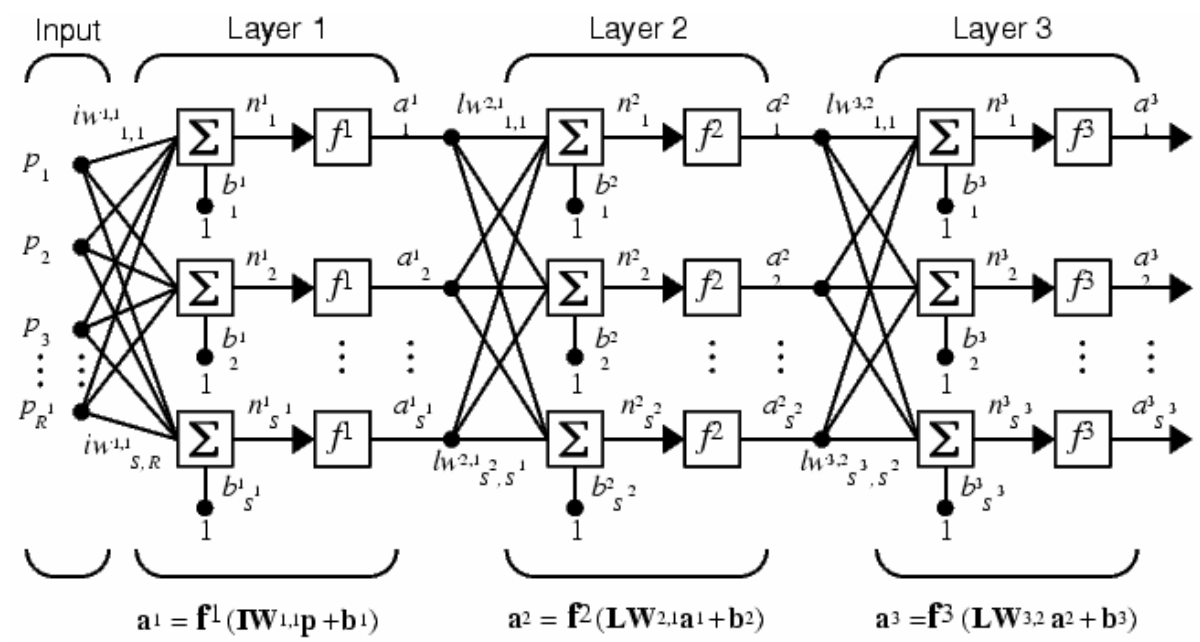

$\mathbf{a}^{3}=\mathbf{f}^{3}\left(\mathbf{L W}^{3,2} \mathbf{f}^{2}\left(\mathbf{L} \mathbf{W}^{2}, \mathbf{f f}^{1}\left(\mathbf{I W}^{1,1} \mathbf{p}+\mathbf{b}^{1}\right)+\mathbf{b}^{2}\right)+\mathbf{b}^{3}\right)$

الشكل (3). يوضح شبكة عصبية متعددة الطبقات

9- مراحل العمل المعتمدة في البحث:

تم اقتراح خوارزمية لأجل اعتماد مصفوفة التواجد لمصفوفة ثنائية حاوية على عناصر صورة بصمة

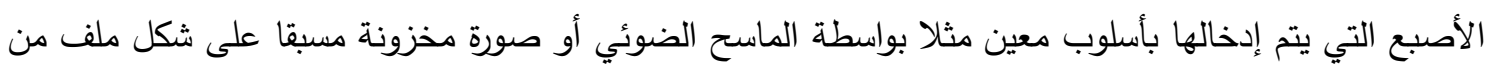
نوع (bmp) بعد أن يتم إجراء بعض التحسينات على الصورة للتخلص من الضوضاء غير المرغوب فيها والتي ملئي 
توجد على حدود البصمة باعتماد الفلتر المتوسط (median filter) وقراءتها كمصفوفة ثنائية رقمية والبرنامج يحدد

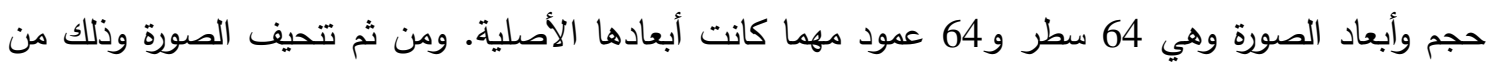
خلال التخلص من الفراغات الموجودة في أعلى وأسفل الصورة و إيجاد مصفوفة التواجد للصورة المحسنة والمختزلة التي أجريت عليها عملية التتحيف وادخلها على الشبكة العصبية لغرض التمييز وممكن تلخيصها بمرحلتين:

المرحلة الأولى:

تم العمل على تدريب شبكة عصبية لأجل التمييز بين مجموعة من عينات بصمات الأصابع حيث تم اعتماد الثبكة العصبية ذات الانتشار العكسي BPNN لغرض التمييز من خلال تهيئة مدخلات الثبكة المتضمنة ملف من نوع bmp والحاوي على خصائص مصفوفة حدوث المشاركة (الطاقة، التباين، التجانس، الارتباط)، أي لئي

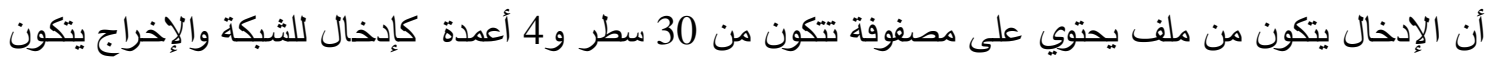

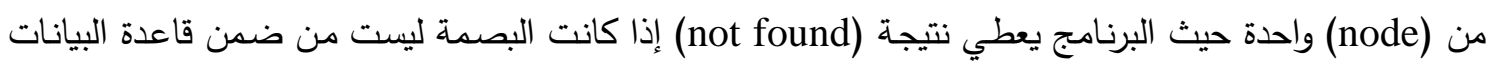

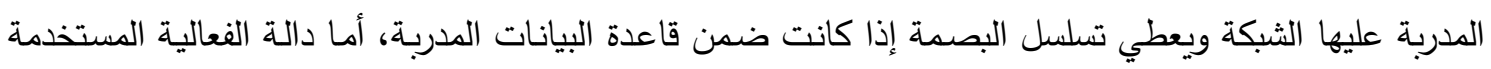
في البرنامج هي الدالة السجماوية (Sigmoid Function) لغرض التمييز بين تلك العينات. المرحلة الثانية:

1- اكتساب صورة بصمة الأصبع من خلال إحدى وسائل الإدخال المتوفرة (مثلا بواسطة الماسح الضوئي أو

صورة مخزونة مسبقا على شكل ملف من نوع (bmp).

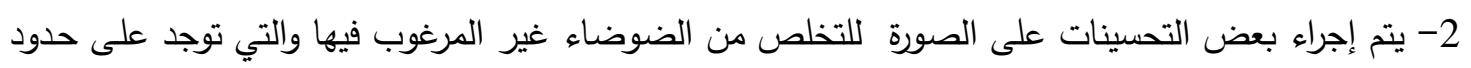

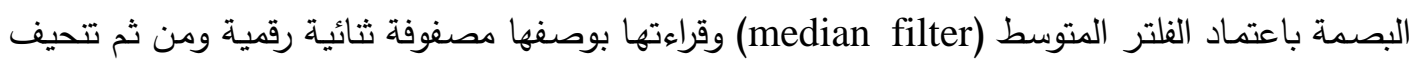

الصورة.

3- حسـاب مصفوفة التواجد للصـورة المحسنة والتي أجريـت عليها عمليـة التتحيف وإدخـال صـفاتها الأربعـة

(الطاقة، التباين، التجانس، الارتباط) على شبكة الانتثار العكسي المدربة مسبقا لأجل التمييز ، حيث البرنامج

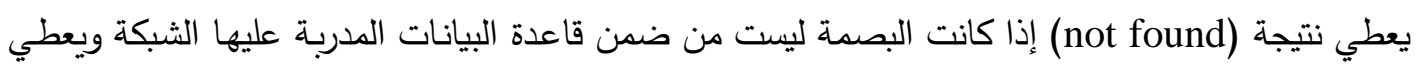
تسلسل البصمة إذا كانت ضمن قاعدة البيانات المدربة كما ذكر سابقا.

10 - 10 - مناقشة النتائج:

من خلال التطبيق العملي للخوارزمية المقترحة تم تدريب الثبكة على عينة تتكون من 30 بصمة أصبع وأثتتت تجارب التدريب للشبكة التوصل إلى استقرارية عالية في التدريب مما دعم مرحلة التمييز عند اختبار صورة بصمة مجهولة. وفيما يلي تطبيق مبسط للخوارزمية:يتم إدخال صورة بصمة أصبع كما في الثكل (4) ومن ثم إجراء بعض التحسينات على الصورة اللتخلص من الضوضاء غير المرغوب فيها والتي توجد على حدود البصمة باعتماد الفلتر المتوسط ( (filter 


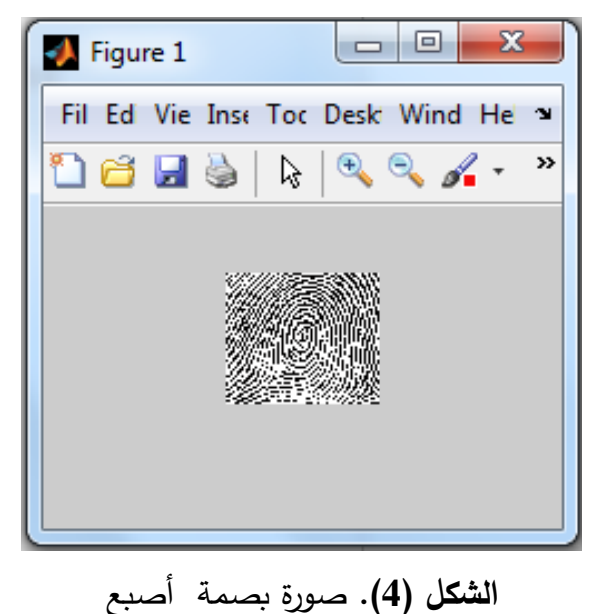

حساب مصفوفة التواجد للصورة المحسنة والتي أجريت عليها عملية التتحيف وإدخال صفاتها الأربعة (الطاقة،

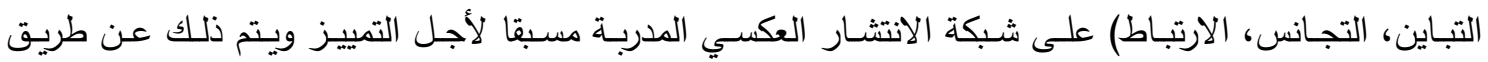
وكما يلي. (graycoprops)

Stats=graycoprops (صورة بصمة الأصبع بعد إجراء بعض التحسين و التتحيف عليها)

Stats $=$

Contrast :207.7400

Correlation:0.0322

Energy:0.0016

Homogeneity:0.1647

نلاحظ نتيجة التدريب إذا كانت الصورة ليست من ضمن قاعدة البيانات الموجودة في الجدول (1) لم يتم

التعرف على الصورة وقد تم طباعة عبارة (not found) أما إذا كانت الصورة من ضمن قاعدة البيانات الموجودة في الجدول (1) فيتم البرنامج بإعطاء تسلسل الصورة ضمن قاعدة البيانات. جدول رقم (1). يمثل قاعدة البيانات الحاوية على تسلسل صور بصمات الأصابع مع قيم الصفات الأربعة لمصفوفة حدوث

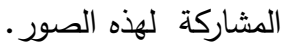

\begin{tabular}{|c|c|c|c|c|}
\hline $\begin{array}{c}\text { التجانس } \\
\text { Homogeneity }\end{array}$ & Energy الطاقة & $\begin{array}{c}\text { الارتباط } \\
\text { correlation }\end{array}$ & Contrast التباين & تسلسل البصمة \\
\hline 0.0999 & $3.6483 \mathrm{e}-004$ & 0.0042 & 729.1510 & $\mathrm{Xc1}$ \\
\hline 0.0944 & $3.3602 \mathrm{e}-004$ & 0.0233 & 800.3683 & $\mathrm{Xc2}$ \\
\hline 0.0905 & $3.7979 \mathrm{e}-004$ & 0.0198 & 874.3388 & $\mathrm{Xc3}$ \\
\hline 0.0242 & $1.0023 \mathrm{e}-005$ & 0.0032 & $3.1934 \mathrm{e}+004$ & Xc4 \\
\hline 0.0240 & $9.4943 \mathrm{e}-006$ & 0.0020 & $3.2004 e+004$ & Xc5 \\
\hline 0.0826 & $4.7192 \mathrm{e}-004$ & 0.0012 & $1.1612 \mathrm{e}+003$ & Xc6 \\
\hline 0.1110 & $6.4433 \mathrm{e}-004$ & 0.0181 & 480.9497 & $\mathrm{xc7}$ \\
\hline 0.0779 & $2.1915 \mathrm{e}-004$ & 0.0033 & $1.4840 \mathrm{e}+003$ & Xc8 \\
\hline 0.0745 & $3.1447 \mathrm{e}-004$ & 0.0288 & $1.6094 \mathrm{e}+003$ & Xc9 \\
\hline 0.0715 & $1.4440 \mathrm{e}-004$ & $4.3454 \mathrm{e}-004$ & $1.8235 \mathrm{e}+003$ & Xc10 \\
\hline 0.0740 & $1.8936 \mathrm{e}-004$ & 0.0059 & $1.5942 \mathrm{e}+003$ & Xc11 \\
\hline 0.0959 & $4.7574 \mathrm{e}-004$ & 0.0035 & 849.2207 & Xc12 \\
\hline 0.0790 & $2.6969 \mathrm{e}-004$ & 0.0108 & $1.3204 \mathrm{e}+003$ & Xc13 \\
\hline 0.0666 & $1.5337 \mathrm{e}-004$ & 0.0059 & $2.1283 \mathrm{e}+003$ & Xc14 \\
\hline 0.0848 & $2.1820 \mathrm{e}-004$ & 0.0039 & $1.2649 \mathrm{e}+003$ & $\mathrm{Xc15}$ \\
\hline
\end{tabular}




\begin{tabular}{|c|c|c|c|c|}
\hline 0.0846 & $3.1878 \mathrm{e}-004$ & 0.0037 & $1.1379 \mathrm{e}+003$ & Xc16 \\
\hline 0.0767 & $3.2227 \mathrm{e}-004$ & 0.0774 & $1.4154 \mathrm{e}+003$ & Xc17 \\
\hline 0.0765 & $2.8313 \mathrm{e}-004$ & 0.0109 & $1.5564 \mathrm{e}+003$ & Xc18 \\
\hline 0.0637 & $1.5916 \mathrm{e}-004$ & 0.0099 & $2.5032 \mathrm{e}+003$ & $\mathrm{Xc19}$ \\
\hline 0.0755 & $2.5310 \mathrm{e}-004$ & $4.7271 \mathrm{e}-004$ & $1.8030 \mathrm{e}+003$ & $\mathrm{Xc20}$ \\
\hline 0.0705 & $2.6781 \mathrm{e}-004$ & 0.0090 & $1.8876 \mathrm{e}+003$ & $\mathrm{Xc21}$ \\
\hline 0.0706 & $1.8529 \mathrm{e}-004$ & 0.0210 & $1.7886 \mathrm{e}+003$ & $\mathrm{Xc22}$ \\
\hline 0.0994 & $7.3099 \mathrm{e}-004$ & 0.0446 & 771.8692 & $\mathrm{Xc23}$ \\
\hline 0.0701 & $3.0618 \mathrm{e}-004$ & $9.2734 \mathrm{e}-004$ & $1.7034 \mathrm{e}+003$ & $\mathrm{Xc24}$ \\
\hline 0.0831 & $2.1911 \mathrm{e}-004$ & 0.0020 & $1.1891 \mathrm{e}+003$ & $\mathrm{Xc25}$ \\
\hline 0.1110 & $6.4433 \mathrm{e}-004$ & 0.0181 & 480.9497 & $\mathrm{Xc26}$ \\
\hline 0.0834 & $4.1894 \mathrm{e}-004$ & 0.0167 & $1.2420 \mathrm{e}+003$ & $\mathrm{Xc27}$ \\
\hline 0.0800 & $3.6298 \mathrm{e}-004$ & 0.0382 & $1.3316 \mathrm{e}+003$ & $\mathrm{Xc28}$ \\
\hline 0.0747 & $2.1598 \mathrm{e}-004$ & 0.0048 & $1.6662 \mathrm{e}+003$ & $\mathrm{Xc29}$ \\
\hline 0.0754 & $2.5947 \mathrm{e}-004$ & 0.0098 & $1.5251 \mathrm{e}+003$ & $\mathrm{Xc30}$ \\
\hline
\end{tabular}

11- الاستنتاجات:

من خلال دراستتا لتمييز صور بصمات الأصـابع تم الاستتناج بأن طريقة حساب مصفوفة التواجد من

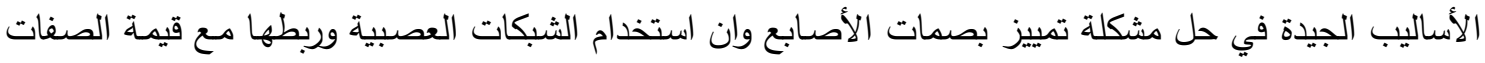
الأربعة لمصفوفة التواجد أضاف قوة أكبر لعملية التمييز، إذ تم تمييز صور بصمة الأصبع وبأحجام مختلفة كذلك

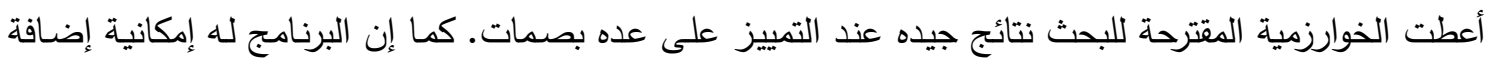

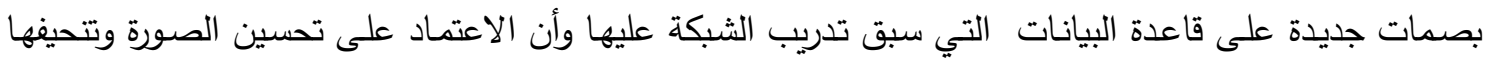
ضمن عملية المعالجة الأولية يؤدي إلى اختزال في وقت التتفيذ لحساب مصفوفة التواجد.

12- الأعمال المستقبلية:

يمكن توظيف أفكار الخوارزمية المقترحة في إمكانية تمييز الوجه والكف أو قزحية العين، إضـافة إلى

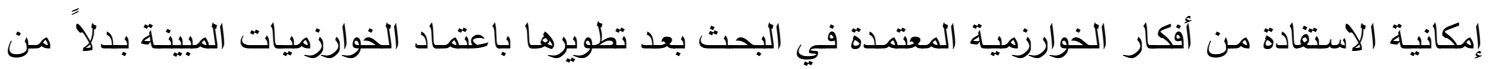
الثبكة العصبية أو استخدام المنطق المضبب الذي من المتوقع أن يقدم تمييز أفضل بسبب ما يمتلك من من إمكانية

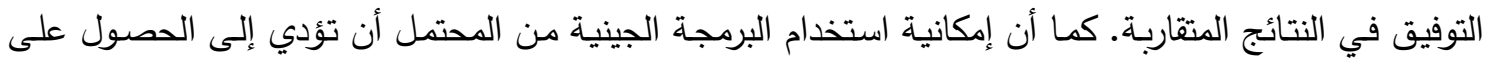
نتائج أدق واقرب إلى الواقع لتمييز بصمة الأصبع. 
المصادر

[1] Abod L.K., (1998), Classification of satellite image using neural network, Ph.D. Thesis, Department of physics, college of science, university of Baghdad.

$$
\begin{aligned}
& \text { شمس، نضير ، خضر ، فوزي (1964). علم البصمات، دراسة تطبيقية شاملة، بيروت. }
\end{aligned}
$$

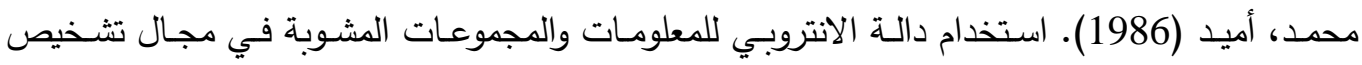

$$
\begin{aligned}
& \text { طبعات الأصابع الجزئية. رسالة ماجستير ، كلية الإدارة والاقتصاد، جامعة بغداد، العراق. } \\
& \text { عوصجي، كامل (1960). فن طبعات الأصابع، الطبعة الرابعة، بغداد. }
\end{aligned}
$$

[5] Chirillo, J. and Blaul, S. (2003), "Implementing Biometric Scurity", April 2003, ISBN:0-7645-2502-6, USA.

[6] Ganapathi, S.k., (2002), "Fingerprint Authentication:Shifting The Electronic Security Paradigm", SC Magazine, January 29.

http://www.scmagazine.com/sconline.htm

[7] Drahanský M., (2005), "Biometric Security System Fingerprints Recognition Technology", Brno University of Technology, Faculty of Information Technology, Department of Intelligent Systems.

[8] Agarwal, M. (2006), "Multi-Impression Enhancement of Fingerprint Images", M.Sc., Thesis, West Virginia University, College of Engineering and Mineral Resources.

سعيد، نغم ثروت، (2007)، "تمييز بصمة الإصبع باعتماد البعد الكسري"، بحث ماجستير ، كلية علوم

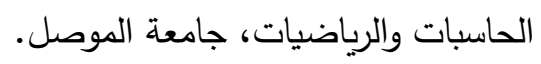

[10] Arati S. Kurani, Dong - HuiXu, Jacob Furst, Daniela Sran Raicu, "Cooccurrence Matrices For Volumetric data", intelligent Multimedia processing Laboratory.

[11] Kiwon Lee, So Hee Jeon, Bynug-Doo Kwan, 2004, "Urban Feature characterization using high-resolution satellite imagery " texture analysis approach.

$$
\text { علام عيسى، الثبكات العصبية الاصطناعية، دار الششرق للنشر، } 2000 .
$$

[13] Mohammed Zeki Kheder, July /13-14/1999, "use of neutral network in Arabic text transliteration", 4th conference on recent trends in computer science, Amman, Jordan.

$$
\text { نضال الأسدي، مقارنة بين شبكتي كوهين والمدرك، جامعة الموصل، العراق، } 2005 .
$$

[15] Rao, V.B. and Rao, H.V., (1993), C++ Neural Networks and Fuzzy Logic, Henry Holt and Company, Ins., New York.

[16] AL_Yaseen Sh., 2000 Face Recognition using Neocognitron Neural Network, M.Sc. Thesis, Department of Computer Engineering , College Of Engineering, University of Mosul.

[17] Wassermann, P.D., (1989), Neural Computing theory and practic van nostrand reinhold: New York.

[18] Jyoti. R \& P.C.Gupta, 2012, "Anew and effective Approach for fingerprint recognition by using feed forward Back propagation Neural network", International Journal of computer Applications (0975_8887). Volume 52, No.10, August. 